\title{
Tissue staining for THSD7A in glomeruli correlates with serum antibodies in primary membranous nephropathy: a clinicopathological study
}

\author{
Shree G Sharma and Christopher P Larsen \\ Arkana Laboratories, Little Rock, AR, USA
}

\begin{abstract}
Thrombospondin type-1 domain-containing 7A (THSD7A) is the most recently recognized target antigen in patients with membranous nephropathy. We stained membranous nephropathy biopsies processed in our laboratory for phospholipase $A_{2}$ receptor and THSD7A over an 18-month period and selected all THSD7Apositive cases for study. Serum samples from most patients were tested by an indirect immunofluorescence assay for the presence of THSD7A antibodies (Euroimmun). A total of 31 patients were diagnosed with THSD7Aassociated membranous nephropathy for a prevalence of $2.4 \%$ among patients with membranous nephropathy. The patients were most often male (male-to-female ratio of 1.6) with a mean age of 62 years and a mean proteinuria of $9.6 \mathrm{~g}$ per day (range 1.1-15.9). Two of the 31 patients had a history of cancer and none were diagnosed with malignancy on follow-up. Serum samples were available at the time of biopsy from 24 patients and all tested positive for antibodies against THSD7A. Conversely, all 20 serum samples from patients with membranous nephropathy who had negative staining for THSD7A were negative for serum reactivity to THSD7A. We conclude that THSD7A tissue staining of kidney biopsies with membranous nephropathy is a sensitive and specific method for the diagnosis of THSD7A-associated membranous nephropathy and it correlates strongly with the serum antibody testing. We also present the clinicopathologic details of the largest cohort to date of THSD7A-associated membranous nephropathy from a single institution.

Modern Pathology (2018) 31, 616-622; doi:10.1038/modpathol.2017.163; published online 15 December 2017
\end{abstract}

Membranous nephropathy is characterized by the renal biopsy finding of subepithelial immune-type deposits and the clinical presentation of nephrotic syndrome. ${ }^{1-3}$ It is a common etiology of nephrotic syndrome in adults, and is the most common glomerulopathy recurring in allografts. ${ }^{3}$ Numerous etiologies are known for this pattern of glomerular immune deposition including autoimmune disease, infection, and cancer, among others. When a known etiology is present, the disease is termed 'secondary'. Those cases without a known associated systemic disease have traditionally been termed 'primary' or 'idiopathic' membranous glomerulopathy. Our understanding of this disease has been transformed with the discovery that most cases of 'idiopathic' membranous nephropathy are due to autoantibodies directed against the phospholipase $\mathrm{A}_{2}$ receptor (PLA2R) protein. ${ }^{4}$ More recently, thrombospondin

Correspondence: Dr SG Sharma, MD, FASN, Arkana Laboratories, 10810 Executive Center Dr., Ste.100, Little Rock, AR 72211, USA. E-mail: Shree.Sharma@arkanalabs.com

Received 17 June 2017; revised 13 September 2017; accepted 14 September 2017; published online 15 December 2017 type-1 domain-containing 7A (THSD7A) protein was described as a second antigenic target of autoantibodies in this disease. ${ }^{5}$ Recent reports have implicated malignancy as the underlying etiology in some cases of THSD7A-associated membranous nephropathy ${ }^{6-8}$ In an effort to further define the clinicopathologic spectrum of THSD7A-associated membranous nephropathy, we undertook the current retrospective study of 31 patients with follow-up of up to 2 years. We also sought to establish the utility of THSD7A staining in renal biopsies and its correlation with antibody titers for the detection of THSD7A-associated membranous nephropathy.

\section{Materials and methods}

All consecutive THSD7A-positive membranous nephropathy from December 2014 to June 2016 accessioned in our laboratory were selected for the study. Patient's medical records were reviewed for clinical data including demographics (age, sex, ethnicity), serum creatinine, serum albumin, proteinuria (24-h urine protein or urine protein to 
creatinine ratio), available laboratory tests, treatment, and follow-up. All renal biopsies were processed using standard protocols for light microscopy, direct immunofluorescence, and electron microscopy. ${ }^{1,9}$ Immunohistochemical staining for THSD7A was performed routinely on all kidney biopsies in which PLA2R was ordered in routine clinical care between December 2014 and June 2016. This included all native kidney biopsies, which showed membranous glomerulopathy seen in our practice during this period with the exception of most cases of membranous lupus nephritis (ISN/RPS Class V). THSD7A stain was interpreted as positive or negative. The stain was called positive if the staining was present in the granular pattern within the subepithelial side of the glomerular basement membranes. Nonspecific staining within the podocytes without a granular pattern was interpreted as negative. All data were collected per the protocol approved by Schulman Associates institutional review board.

\section{Standard Renal Biopsy Processing Techniques}

All light microscopy samples were stained with hematoxylin and eosin, Jones methenamine silver, Masson trichrome, and periodic acid-Schiff reagent. ${ }^{9}$ All direct immunofluorescence sections were cut at $4 \mu \mathrm{m}$ and reacted with fluorescein-tagged polyclonal rabbit anti-human antibodies to IgG, IgA, IgM, C3, C1q, fibrinogen, and $\kappa$ - and $\lambda$-light chains (Dako, Carpenteria, CA, USA) for $1 \mathrm{~h}$, rinsed, and a coverslip applied using the aqueous mounting media. For electron microscopy, thin sections were examined in a Jeol JEM-1011 electron microscope (Jeol, Tokyo, Japan). Photomicrographs were routinely taken at $\times 5,000 \times 12,000$, and $\times 20,000$ magnifications.

\section{PLA2R1 and THSD7A Staining}

The THSD7A immunohistochemical stain was performed on a Dako Autostainer Link 48 as previously described. ${ }^{1}$ In brief, THSD7A was detected in paraffin-embedded sections by immunoperoxidase staining using anti-THSD7A antibodies (SigmaAldrich) at a dilution of 1:800 (Figure 1). The PLA2R1 immunofluorescence staining procedure was performed as previously described on formalin-fixed paraffin-embedded tissue. ${ }^{10}$

\section{Clinical Definitions}

Complete remission and partial remission was defined per the KDIGO guidelines. ${ }^{11}$ Complete remission was defined as urinary protein excretion less than $0.3 \mathrm{~g} / \mathrm{d}$ (uPCR $<300 \mathrm{mg} / \mathrm{g}$ or $30 \mathrm{mg} / \mathrm{mmol}$ ), confirmed by 2 values at least 1 week apart, accompanied by normal serum albumin and normal serum creatinine. Partial remission was defined as

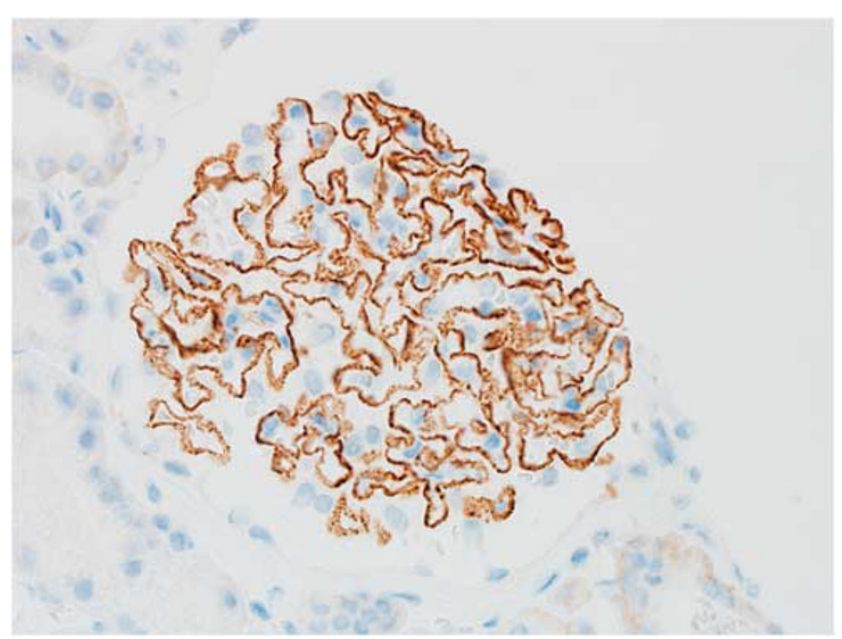

Figure 1 Strong global granular capillary wall staining for THSD7A in a patient of THSD7A-associated membranous glomerulopathy (original magnification $\times 400$ ).

urinary protein excretion less than $3.5 \mathrm{~g} / \mathrm{d}$ (uPCR $<3500 \mathrm{mg} / \mathrm{g}$ or $350 \mathrm{mg} / \mathrm{mmol}$ ), and a $50 \%$ or greater reduction from peak value; confirmed by 2 values at least 1 week apart, accompanied by improvement or normalization of serum albumin and stable serum creatinine.

\section{Serum testing for THSD7A Antibodies}

Circulating autoantibodies to PLA2R and THSD7A were detected using indirect immunofluorescence assays (EUROIMMUN, Lübeck, Germany). Each of the slides contained two different biochips in one incubation field. One biochip was coated with human embryonic kidney (HEK)293 cells that express the PLA2R or THSD7A protein, whereas the second biochip, which serves as a negative control, contained non-transfected HEK293 cells. Serum diluted 1:10 was applied to the slides and incubated for $30 \mathrm{~min}$. A FITC-conjugated anti-human IgG-antibody was used for detection of bound IgGantibodies. Cytoplasmic fluorescence of the transfected cells only at this dilution was considered to be positive. Cases that tested positive at a titer of 1:10 were then tested at dilutions of 1:100, 1:500, and 1:1000.

\section{Results}

A total of 31 of the 1318 membranous nephropathy cases diagnosed in our laboratory during the study period were positive for THSD7A $(2.4 \%)$. Nine of these patients were previously reported. ${ }^{1}$ Among these 31 patients there were 19 males and 12 females (male-to-female ratio of 1.6). The mean age was 62 years (range 17-91 years). Among the patients with known ethnicity $(n=25)$ the disease was more common in Caucasians $(n=14)$, followed by 
African-Americans $(n=6)$, Hispanics $(n=3)$, and Native Indians $(n=2)$. Nephrotic syndrome was the most common presentation. Twenty-eight (28) patients presented with full nephrotic syndrome, two of which also had acute kidney injury and three patients presented with non-nephrotic range proteinuria, one of which had subacute renal failure. The mean proteinuria was $9.62 \mathrm{~g}$ (range 1.1-15.92, $n=28)$. The mean serum albumin was $2.05 \mathrm{~g} / \mathrm{dl}$ (range $0.5-3.2, n=24$ ). The mean serum creatinine was $1.4 \mathrm{mg} / \mathrm{dl}$ (range $0.6-5, n=30$ ). The clinical and laboratory data are presented in Table 1. On renal biopsy, all the cases had positive THSD7A and two cases were also positive for PLA2R.

The renal biopsies were evaluated for light microscopy, immunofluorescence, and electron microscopy. On light microscopy, the mean number of open glomeruli $(n=30)$ were 29.4 (range 12-58), globally sclerotic glomeruli were 4.1 (14\%) ((range: 0-14; percentage: 0-39\%). In addition, five cases had segmental glomerulosclerosis and one patient had nodular diabetic glomerulosclerosis. No tubulointerstitial scarring was seen in $11(35.5 \%)$ patients. Tubulointerstitial scarring was mild $(\leq 25 \%)$ in 9 $(29 \%)$, moderate $(26-50 \%)$ in $6(19.4 \%)$ and severe $(>50 \%)$ in five $(16 \%)$ patients. No vascular disease was seen in $11(35.5 \%)$ patients. Arteriosclerosis was mild $(\leq 25 \%)$ in $6(19.4 \%)$, moderate $(26-50 \%)$ in 9 $(29 \%)$ and severe in $5(16 \%)$ patients. Immunofluorescence was positive for only IgG, C3, kappa and lambda light chains in 30 cases and one case showed full-house pattern of staining. Electron microscopy in all the cases showed subepithelial deposits. One case showed segmental mesangial deposits (3\%) and there were no cases with subendothelial deposits or tubuloreticular inclusions. Ehrenreich-Churg staging $(n=26)$ on electron microscopy was as follows: stage 1,10 cases; stage 2, 8 cases; stage 3,7 cases; and stage 4,1 case.

Follow-up data were available in 29 patients with a mean follow-up of 12 months (range 1-25 months). The mean proteinuria on follow-up was $6.9 \mathrm{~g}$ (range $0-20.97 ; n=25$ ) and in one patient urinalysis showed $3+$ protein, mean serum creatinine was $1.8 \mathrm{mg} / \mathrm{dl}$ (range 0.6-7.5; $n=29$ ) and mean serum albumin was $2.8 \mathrm{~g} / \mathrm{dl}$ (range $1-4.1 ; n=27$ ). A total of 24 patients had complete treatment and follow-up data available. In the total group, there were four patients who underwent complete remission and all were treated with immunosuppression. Four of the five cases $(80 \%)$ that underwent partial remission were treated with immunosuppression and the remaining case with angiotensin blockade alone. Fifteen cases had no remission, among which $10(67 \%)$ were treated with immunosuppression and the remaining received angiotensin blockade alone.

Serum samples from 24 patients were available at the time of diagnosis and all of them were positive (titer 1:10-1:500) for THSD7A antibodies. Serum samples from 20 patients with biopsy proven THSD7A-negative membranous nephropathy were
Table 1 Summary of clinical and laboratory findings of patients with THSD7A-associated membranous glomerulopathy

Clinical features
Gender (male:female)

Mean age at biopsy, years (range)

Race

Caucasian

African-American

Hispanic

Native Indian

Unknown

Laboratory data

Proteinuria at the time of biopsy, g/24 h,

$n=28$

Proteinuria at the time of follow-up, g/24 h, $n=25$

Serum albumin at the time of biopsy, g/dl, $n=24$

Serum albumin at the time of follow-up, g/dl, $n=27$

Serum creatinine at the time of biopsy, $\mathrm{mg} / \mathrm{dl}, n=30$

Serum creatinine at the time of follow-up, $\mathrm{mg} / \mathrm{dl}, n=29$

Serology test results

Positive ANA

Decreased complements (low)

Positive rheumatoid factor

$\mathrm{M}$-spike on serum protein electrophoresis

Associated conditions

Hypertension

Diabetes mellitus

Systemic lupus erythematosus

Gout

Malignancy
19:12

62 (17-91)

n (\%)

$14(45.2)$

6 (19.4)

$3(9.6)$

2 (6.5)

6 (19.4)

Mean (range)

9.62 (1.1-

15.92)

$6.9(0.0-20.97)$

$2.05(0.5-3.2)$

$2.8(1-4.1)$

$1.4(0.6-5)$

$1.8(0.6-7.5)$

$\mathrm{n}(\%)$

$2(7 \%)$

$1(3 \%)$

$2(7 \%)$

$1(3 \%)$

$20(65 \%)$

$9(29 \%)$

$1(3 \%)$

$1(3 \%)$

$2(6 \%)$ negative for serum reactivity to THSD7A. The THSD7A antibody titer did not correlate with the level of proteinuria and was not predictable of disease remission or response. Ten patients had titer of $1: 10$ with an average proteinuria of $10.1 \mathrm{~g}$ (range 2.8-23 g). Follow-up proteinuria was available for nine patients in this group and no patient underwent complete remission, one had partial remission and eight had no remission. Six of the patients in this group were treated with immunosuppression and three received angiotensin blockade alone. Eleven patients had a titer of 1:100 with an average proteinuria of $10.5 \mathrm{~g}$ (range $3-15 \mathrm{~g}$ ). In this group, seven patients had an available follow-up proteinuria and two patients underwent complete remission, two partial remission, and three had no remission. Six of the patients with follow-up were treated with immunosuppression and one patient received angiotensin blockade alone. Three patients had titer of 1:500 at the time of diagnosis with an average proteinuria of $9.2 \mathrm{~g}$ (range $2.4-15.9 \mathrm{~g}$ ). None of these three underwent remission. Two of these patients were treated with immunosuppression and 


\begin{tabular}{|c|c|c|c|c|c|c|c|c|c|c|c|}
\hline \multirow[b]{2}{*}{ Serial No. } & \multirow[b]{2}{*}{ Age } & \multicolumn{4}{|c|}{ Time of biopsy data } & \multirow[b]{2}{*}{ Treatment } & \multicolumn{5}{|c|}{ Time of follow-up data } \\
\hline & & $\begin{array}{l}\text { THSD7A } \\
\text { titer }\end{array}$ & $\begin{array}{l}\text { Proteinuria } \\
\text { (g/day) }\end{array}$ & $\begin{array}{l}\text { Serum } C r \\
(\mathrm{mg} / \mathrm{dl})\end{array}$ & $\begin{array}{l}\text { Serum } \\
\text { albumin } \\
(\mathrm{g} / \mathrm{dl})\end{array}$ & & $\begin{array}{l}\text { F/u (in } \\
\text { months) }\end{array}$ & $\begin{array}{l}\text { Serum } C r \\
(\mathrm{mg} / \mathrm{dl})\end{array}$ & $\begin{array}{l}\text { Proteinuria } \\
\text { (g/day) }\end{array}$ & $\begin{array}{l}\text { Serum } \\
\text { albumin } \\
(\mathrm{g} / \mathrm{dl})\end{array}$ & $\begin{array}{l}\text { THSD7A } \\
\quad \text { titer }\end{array}$ \\
\hline 1 & 61 & $1: 10$ & 7 & 1.2 & 1.4 & $\begin{array}{l}\text { Cyclophosphamide, Prednisone, } \\
\text { Cyclosporine }\end{array}$ & 20 & 1.84 & 8.04 & 1 & NA \\
\hline 2 & 43 & $1-10$ & 12.3 & 0.9 & 1 & NA & NA & NA & NA & NA & NA \\
\hline 3 & 91 & $1-10$ & 8 & 0.9 & 2.5 & Hospice & 6 & 0.96 & 20 & 2.2 & NA \\
\hline 4 & 66 & $1-10$ & 8.6 & 0.8 & $\mathrm{NA}$ & $\begin{array}{l}\text { Tacrolimus, Repository } \\
\text { corticotropin injection }\end{array}$ & 13 & 1.1 & 3.3 & 2.6 & NA \\
\hline 5 & 63 & $1-10$ & 10 & 1.21 & 2 & $\begin{array}{l}\text { Cyclosporine, Prednisone, } \\
\text { Cyclophosphamide }\end{array}$ & 12 & 1.4 & 12.5 & 2.4 & NA \\
\hline 6 & 56 & $1-10$ & 7.1 & 1 & NA & Prednisone, Cyclosporine, ACEi & 12 & 1.3 & 5.03 & 2.6 & NA \\
\hline 7 & 47 & $1-10$ & 15.1 & 0.6 & NA & ACEi & 7 & 0.6 & 8.84 & 2.8 & NA \\
\hline 8 & 75 & $1-10$ & 23 & 1.6 & 1.9 & Cyclophosphamide, Prednisone & 6 & 0.9 & 6.6 & 2.6 & NA \\
\hline 9 & 64 & $1: 10$ & 2.8 & 1.5 & 2.9 & Rituximab, ACEi & 8 & 1.43 & 1.7 & 3.6 & NA \\
\hline 10 & 55 & $1: 10$ & 7.1 & 0.7 & 2.7 & ACEi & 7 & 0.8 & 10 & NA & NA \\
\hline 11 & 58 & $1-100$ & 10.1 & 1 & 1.6 & $\begin{array}{l}\text { Repository corticotropin } \\
\text { injection, Predniosne }\end{array}$ & 25 & 1.3 & 0 & 4.1 & Neg \\
\hline 12 & 54 & $1-100$ & 15 & 0.8 & 1.9 & $\begin{array}{l}\text { Cyclosphosphamide, } \\
\text { Cyclosporine, Prednisone, ACEi }\end{array}$ & 24 & 0.87 & 4 & 3.5 & NA \\
\hline 13 & 76 & $1-100$ & 10.7 & 1.4 & 2 & Cyclosporine, Tacrolimus & 22 & 1.4 & 11 & 2 & NA \\
\hline 14 & 56 & $1-100$ & 3 & 1.3 & low & $\mathrm{ARB}$ & 20 & 1.06 & 0.42 & 3.6 & NA \\
\hline 15 & 73 & $1-100$ & 10.5 & 2.3 & 2.5 & Rituximab & 12 & 2.4 & NA & 3.8 & NA \\
\hline 16 & 57 & $1-100$ & 15 & 1.2 & 2.5 & Cyclosporine, Prednisone, ACEi & 10 & 1.12 & 12.6 & 2.5 & NA \\
\hline 17 & 74 & $1-100$ & 12 & 1 & NA & Cyclosporine, Prednisone & 9 & 1.3 & 0.3 & 3.5 & NA \\
\hline 18 & 70 & $1-100$ & 12 & 0.88 & 1.8 & Cyclophosphamide, Prednisone & 7 & 0.68 & 2.7 & 3.2 & NA \\
\hline 19 & 17 & $1: 100$ & 6.4 & 0.57 & 1.8 & Prednisone, ACEi & 3 & 0.58 & NA & 1.2 & NA \\
\hline 20 & 63 & $1: 100$ & 10 & 2.4 & 2 & Mycophenolic acid & 8 & 3.04 & $3+$ & 2.5 & NA \\
\hline 21 & 25 & $1: 100$ & $3+$ & 0.62 & 1.6 & $\begin{array}{l}\text { Prednisone, Mycophenolic acid, } \\
\text { Plaquenil }\end{array}$ & 1 & 0.6 & NA & 1.3 & NA \\
\hline 22 & 70 & $1-500$ & 2.4 & 1.9 & 2.8 & $\begin{array}{l}\text { Cyclophosphamide, } \\
\text { Cyclosporine, Mycophenolic } \\
\text { acid }\end{array}$ & 13 & 2.8 & 2 & 2.6 & NA \\
\hline 23 & 67 & $1: 500$ & $3+$ & 0.73 & 2.2 & ACEi & 6 & 0.81 & 11 & 2.1 & NA \\
\hline 24 & 63 & $1: 500$ & 15.92 & 3.67 & 0.5 & Prednisone, Cyclophosphamide & 7 & 7.5 & 20.97 & $<1.5$ & NA \\
\hline 25 & 64 & NA & 1.1 & 0.6 & 3.2 & Prednisone, Cyclosporine & 25 & 1.08 & 0 & 4 & NA \\
\hline 26 & 76 & NA & 12.4 & 3.3 & 1.8 & $\begin{array}{l}\text { Cyclophosphamide and } \\
\text { Prednisone }\end{array}$ & 24 & 3.3 & 1.3 & 3.7 & Neg \\
\hline 27 & 67 & NA & $>3.5$ & 5 & 3.2 & Dialysis & 16 & 8 & NA & 4.1 & NA \\
\hline 28 & 70 & NA & 14 & 2.3 & NA & $\begin{array}{l}\text { Cyclophosphamide, Prednisone, } \\
\text { ACEi }\end{array}$ & 15 & 1.7 & 4 & 2.6 & NA \\
\hline 29 & 70 & NA & 6 & 1.1 & 1.9 & Rituximab, ACEi & 8 & 1.09 & 0.31 & 4 & NA \\
\hline 30 & 71 & NA & NA & NA & NA & Consult case, no follow-up & NA & NA & NA & NA & NA \\
\hline 31 & 66 & NA & 8.6 & 0.9 & 1.6 & ACEi & 2 & 1 & 6 & NA & NA \\
\hline
\end{tabular}


one patient received angiotensin blockade alone. Treatment regimens employed were heterogeneous.

Follow-up serum samples after treatment were available from two patients, both of which were negative for THSD7A antibodies and the patients had undergone remission. The first patient was a 58-yearold Caucasian male who presented with NS (10.1 g proteinuria, serum albumin $1.6 \mathrm{~g} / \mathrm{dl}$ and $\mathrm{Cr}-1 \mathrm{mg} / \mathrm{dl}$ ) and had no tubular atrophy and interstitial fibrosis on renal biopsy. At the time of diagnosis, the patient's THSD7A antibody titer was 1:100 and on follow-up after 2 years it was negative after being treated with repository corticotropin injection and prednisone. The patient had no proteinuria, albumin was $4.0 \mathrm{~g} / \mathrm{dl}$ and creatinine was $1.3 \mathrm{mg} / \mathrm{dl}$ at the time of follow-up. The second patient in our series was 76-year-old African-American male who also presented with NS (12.4 g proteinuria, serum albumin $1.8 \mathrm{~g} / \mathrm{dl}$, and $\mathrm{Cr} 3.3 \mathrm{mg} / \mathrm{dl})$. The renal biopsy at the time of diagnosis showed a membranous nephropathy with positive staining for THSD7A and negative staining for PLA2R and a background of moderate interstitial fibrosis and severe vascular disease. The patient received cyclophosphamide and steroids (Ponticelli regimen) and serum testing performed 6 months after the treatment initiation was negative for THSD7A antibodies. Follow-up at 24 months after the biopsy showed a reduction in the patient's proteinuria to $1.3 \mathrm{~g}$, serum albumin was $3.7 \mathrm{~g} / \mathrm{dl}$, and creatinine was $3.3 \mathrm{mg} / \mathrm{dl}$.

The treatment of the patients in our series was variable ranging from cyclophosphamide with steroids, Rituximab, cyclosporine, steroids, mycophenolic acid to ACE inhibitors only. One patient reached end stage kidney disease, in which there was severe tubular atrophy and interstitial fibrosis at the time of diagnosis. The THSD7A titer, proteinuria, serum albumin and creatinine levels at the time of presentation and follow-up along with the treatment is summarized in Table 2.

\section{Discussion}

Membranous nephropathy is a pattern of glomerular immune complex disease with a variety of etiologies. ${ }^{10}$ This disease has seen marked changes in our understanding, diagnosis, and treatment in the past 15 years. Based on the Heymann nephritis model of membranous nephropathy, it was long been suspected that membranous nephropathy resulted from autoantibodies directed against podocyte antigens. $^{12}$ The discovery of neutral endopeptidase in antenatal membranous nephropathy was the first human confirmation of this model. ${ }^{13}$ The subsequent identification of PLA2R as the antigenic target in most cases of primary membranous nephropathy provided further evidence and has led to rapid changes in the area of treatment and diagnosis of the disease. ${ }^{4}$ Specifically, among patients with PLA2R- associated membranous nephropathy, it is now possible to select patients who are most likely to benefit from treatment and to recognize patients who might undergo spontaneous remission prior to treatment. ${ }^{14}$ THSD7A-associated membranous nephropathy is a more recently recognized subtype of membranous nephropathy and the clinicopathologic significance of this form of membranous nephropathy is still being uncovered. We provide data here about the clinicopathologic characteristics in a large cohort of THSD7A-associated membranous nephropathy from a single laboratory and show that THSD7A staining on the renal biopsy is highly specific for the detection of THSD7A-associated membranous nephropathy and it correlates with serologic positivity of THSD7A antibodies.

A recently large case series gathering patients from multiple institutions reported clinicopathologic characteristics in 40 patients with THSD7Aassociated membranous nephropathy. ${ }^{7}$ The prevalence of THSD7A-associated membranous nephropathy in a subset of these patients was found to be $2.6 \%$, which is similar to the prevalence of $2.4 \%$ among patients with membranous nephropathy in our laboratory. Many of the clinical and pathologic details are similar between these cohorts including the mean patient age, degree of proteinuria, and the lack of correlation between the proteinuria and THSD7A antibody levels. However, we found that the patient gender was more often male (61\%) compared to $43 \%$ in the Hoxha, et $a 7^{7}$ cohort. This male gender predominance seen in our cohort is similar to that which is described in case series detailing PLA2R-associated membranous nephropathy. ${ }^{10,15}$ Another important discrepancy is the relatively low association with malignancy in our cohort at only $6 \%$ compared with $20 \%$ reported by Hoxha, et $\mathrm{al}^{7}$ There were no patients found to have been diagnosed with malignancy on follow-up. The two patients in our series who were diagnosed with cancer included one patient who underwent lumpectomy and radiation therapy for breast carcinoma (Stage1b) 3 years prior to diagnosis of membranous nephropathy as well as a history of thyroidectomy for cancer $>30$ years prior. The second patient had previously been treated for renal cell carcinoma as well as breast carcinoma. Many different types of cancer have been shown to have some degree of positive immunohistochemical staining for THSD7 ${ }^{6}$ and a previous case report makes a strong argument for the connection between THSD7Aassociated membranous nephropathy and malignancy. ${ }^{8}$ In this report, the patient's tumor was found to be positive for THSD7A on immunohistochemical analysis and have increased copy number of the THSD7A gene. In addition, the patient's THSD7A antibodies responded to treatment of the malignancy. Nevertheless, the present case series suggests that malignancy might not be as common as previously reported in patients with THSD7Aassociated membranous nephropathy. Additional 
studies with larger cohorts and longer follow-up are needed to definitively determine the association of cancer with THSD7A-associated membranous nephropathy.

The role of serum testing for anti-PLA2R antibody levels have become important in treating and monitoring the therapy of patients with PLA2Rassociated membranous nephropathy in recent years. ${ }^{14}$ More data are needed to determine the clinical utility of similar testing for serum THSD7A antibody testing. However, this information will be more difficult to obtain than PLA2R data based on the relative rarity of this subtype of the disease. For this case series, serum samples were available at the time of biopsy on 24 patients with membranous nephropathy and positive THSD7A staining. All 24 of these patients tested positive for antibodies against THSD7A. We have also tested 20 serum samples from patients with membranous nephropathy that was negative for THSD7A staining on biopsy and found that all were negative for the presence of THSD7A serum antibodies. These findings demonstrate the high sensitivity and specificity of tissue staining for THSD7A for the recognition of THSD7Aassociated membranous nephropathy. In a previous report, ${ }^{1}$ we identified two patients with dual positivity for both THSD7A and PLA2R both on the biopsy staining and in the serum antibody testing. The larger cohort presented here failed to identify any additional cases with dual positivity, suggesting that this phenomenon is uncommon. Follow-up serum samples after treatment were available from two patients, both of which were negative for THSD7A antibodies. This correlated with clinical improvement and a significant reduction in the degree of proteinuria in both patients. For PLA2Rassociated membranous nephropathy, it has been shown that high titer antibodies are associated with poor prognosis and response to therapy. ${ }^{2,15}$ Low-titer THSD7A antibodies (1:10) at the time of diagnosis did not correlate with the level of proteinuria and were not predictable of disease remission or response in our cohort. Three patients had high titers (1:500) of THSD7A antibodies and none showed clinical response. However, based on the small sample sizes in each of these titer groups, it is premature to make any definitive conclusions about the relationship between the titers and treatment response. In the total group, there were four patients who underwent complete remission and all were treated with immunosuppression. We did not identify any patients who underwent spontaneous remission during the relatively short follow-up period.

We report the largest case series of THSD7Aassociated membranous nephropathy from a single institution and demonstrate that tissue staining for THSD7A is highly sensitive and specific for the detection of THSD7A-associated membranous nephropathy and it correlates strongly with serum positivity. This series also provides additional clinicopathologic information in patients with this type of membranous nephropathy. Importantly, we find a smaller proportion of patients with an association with malignancy than has previously been reported. ${ }^{7}$ The shortcomings of our study are the limited follow-up of only 2 years and lack of follow-up serological tests to evaluate antibody titers in most patients.

\section{Acknowledgments}

We thank I Shin Wen for her assistance in collecting the clinical histories.

\section{Disclosure/conflict of interest}

The authors declare no conflict of interest.

\section{References}

1 Larsen CP, Cossey LN, Beck LH. THSD7A staining of membranous glomerulopathy in clinical practice reveals cases with dual autoantibody positivity. Mod Pathol 2016;29:421-426.

2 Cattran DC, Brenchley PE. Membranous nephropathy: integrating basic science into improved clinical management. Kidney Int 2017;91:566-574.

3 Ronco P, Debiec H. Pathophysiological advances in membranous nephropathy: time for a shift in patient's care. Lancet 2015;385:1983-1992.

4 Beck LH Jr, Bonegio RG, Lambeau G, et al. M-type phospholipase A2 receptor as target antigen in idiopathic membranous nephropathy. $N$ Engl J Med 2009;361:11-21.

5 Tomas NM, Beck LH Jr, Meyer-Schwesinger C, et al. Thrombospondin type-1 domain-containing $7 \mathrm{~A}$ in idiopathic membranous nephropathy. N Engl J Med 2014;371:2277-2287.

6 Stahl PR, Hoxha E, Wiech T, et al. THSD7A expression in human cancer. Genes Chromosomes Cancer 2017;56: 314-327.

7 Hoxha E, Beck LH Jr, Wiech T, et al. An indirect immunofluorescence method facilitates detection of thrombospondin type 1 domain-containing 7A-specific antibodies in membranous nephropathy. J Am Soc Nephrol 2017;28:520-531.

8 Hoxha E, Wiech T, Stahl PR, et al. A mechanism for cancer-associated membranous nephropathy. N Engl J Med 2016;374:1995-1996.

9 Walker PD, Cavallo T, Bonsib SM et al. Practice guidelines for the renal biopsy. Mod Pathol 2004;17: 1555-1563.

10 Larsen CP, Messias NC, Silva FG, et al. Determination of primary versus secondary membranous glomerulopathy utilizing phospholipase A2 receptor staining in renal biopsies. Mod Pathol 2013;26:709-715.

11 Kidney Disease: Improving Global Outcomes (KDIGO) Glomerulonephritis Work Group. KDIGO Clinical Practice Guideline for Glomerulonephritis. Kidney Int Suppl 2012;2:139-274.

12 Heymann W, Hackel DB, Harwood S, et al. Production of nephrotic syndrome in rats by Freund's adjuvants 
and rat kidney suspensions. Proc Soc Exp Biol Med 1959;100:660-664.

13 Debiec H, Guigonis V, Mougenot B, et al. Antenatal membranous glomerulonephritis due to anti-neutral endopeptidase antibodies. N Engl J Med 2002;346: 2053-2060.

14 De Vriese AS, Glassock RJ, Nath KA, et al. A proposal for a serology-based approach to membranous nephropathy. J Am Soc Nephrol 2017;28: 421-430.

15 Hoxha E, Thiele I, Zahner G, et al. Phospholipase A2 receptor autoantibodies and clinical outcome in patients with primary membranous nephropathy. J Am Soc Nephrol 2014;25:1357-1366. (c) (i) $(\theta)$ This work is licensed under a Creative Commons Attribution-NonCommercialNoDerivs 4.0 International License. The images or other third party material in this article are included in the article's Creative Commons license, unless indicated otherwise in the credit line; if the material is not included under the Creative Commons license, users will need to obtain permission from the license holder to reproduce the material. To view a copy of this license, visit http:/creativecommons.org/licenses/ by-nc-nd/4.0/

(C) The Author(s) 2018 